\title{
Estradiol/Testosterone Imbalance: Impact on Coronary Heart Disease Risk Factors in Postmenopausal Women
}

\author{
Wen Dai Yan Li Hongyun Zheng \\ Department of Clinical Laboratory, Renmin Hospital of Wuhan University, Wuhan, China
}

For editorial comment see p. 247

\section{Key Words}

Estradiol/testosterone ratio - Coronary heart disease •

Postmenopausal women

\begin{abstract}
Objective: Several groups have reported the important role of the estradiol/testosterone $\left(E_{2} / T\right)$ ratio in benign prostatic hyperplasia and cerebral vessels. However, there has been no study on the role of the $\mathrm{E}_{2} / \mathrm{T}$ ratio in women with coronary heart disease (CHD). This study aimed to evaluate the association among the ratio of sex hormones and known risk factors of atherosclerosis in postmenopausal women with CHD. Methods: 114 controls and 124 postmenopausal women with CHD were selected for this study. Serum levels of estradiol, testosterone, aromatase, sex hormone-binding globulin (SHBG), lipid-lipoprotein profile and high-sensitivity C-reactive protein were determined. Results: Compared with the control, the $E_{2} / T$ ratio decreased from $5.35 \pm 2.78$ to 3.88 \pm 2.51 ( $p<0.0001)$. Multiple linear regression analysis showed that the $E_{2} / T$ ratio was negatively associated with total cholesterol, low-density lipoprotein cholesterol (LDL-c) and the atherogenic index of plasma, but positively associated with high-density lipoprotein cholesterol (HDL-c) and HDL-c/LDL-c (for all, $p<0.0001$ ). We found that there was a negative correlation between the $E_{2} / T$ ratio and aromatase
\end{abstract}

$(r=-0.192, p=0.032)$ and a positive correlation between aromatase and SHBG $(r=0.938)$. Conclusion: The balance of the serum $\mathrm{E}_{2} / \mathrm{T}$ ratio was broken in the women with $\mathrm{CHD}$, and an imbalanced $E_{2} / T$ ratio showed a strong association with cardiovascular risk factors in postmenopausal women with CHD.

Copyright $\odot 2012$ S. Karger AG, Basel

\section{Introduction}

Prior to menopause, women have a much lower risk of coronary heart disease (CHD) compared with men of the same age; however, menopause initiates a phase of increased risk [1]. This indicates that sex hormones play a role in the development of CHD.

Sex steroid deficiency is considered a crucial factor responsible for the menopause-associated changes in the CHD risk factor profile, and consequently for the increase in CHD risk [2]. Although increasing evidence supports an association between endogenous sex hormones and cardiovascular disease, the results still remain controversial. Several studies have shown a positive relationship between estrogen and high-density lipoprotein cholesterol (HDL-c) and a negative relationship with lowdensity lipoprotein cholesterol (LDL-c) [3-5], while other

\section{KARGER \\ Fax +41613061234 \\ E-Mail karger@karger.ch}

www.karger.com (c) 2012 S. Karger AG, Basel

$0008-6312 / 12 / 1214-0249 \$ 38.00 / 0$

Accessible online at:

www.karger.com/crd 
studies conclude that estradiol levels are not associated with a risk of CHD [6-8]. Studies on androgens are also conflicting. Some studies found that higher levels of androgens and sex hormone-binding globulin (SHBG) were associated with a reduced level of atherosclerosis [9-10]; however, others found a positive association between testosterone (the main form of androgen) and cardiovascular disease risk $[11,12]$. Owing to these controversial results, we consider that it may not be enough to investigate the association between a single hormone and CHD risk factors. Therefore, we should combine estrogens and androgens together, i.e. the estradiol/testosterone $\left(\mathrm{E}_{2} / \mathrm{T}\right) \mathrm{ra}-$ tio, to further study the association between sex hormones and CHD risk factors.

To explore this option, we hypothesized that an imbalance of estradiol and testosterone may be an important factor leading to the development of CHD in postmenopausal women. In this study, we first want to measure the levels of the serum sex hormones in both normal control and CHD cases, and to observe whether it is imbalanced in cases with $\mathrm{CHD}$, and then to explore further the relationship between imbalanced sex hormones and CHDrelated risk factors in postmenopausal women with CHD.

\section{Methods}

\section{Subjects}

Subjects were recruited from the Department of Cardiology at the Renmin Hospital of Wuhan University. This study was part of a survey aiming to explore the best physiological $\mathrm{E}_{2} / \mathrm{T}$ ratio in postmenopausal women. For this purpose 2,000 postmenopausal women (aged 50-75 years) were recruited during the time period from September 2010 to March 2011. All subjects underwent coronary angiography. Individuals found to have $\geq 70 \%$ occlusion of at least one major coronary artery were defined as CHD (cases); those with completely normal coronary arteries and with $\leq 50 \%$ occlusion in all coronary arteries (free of significant stenosis) constituted the control group [13]. The subjects had no other serious illnesses and were neither current or ever-users of hormone replacement treatment or ovariectomy. All subjects gave informed consent, and were excluded from the present analysis if they were taking cholesterol-lowering medications. Of the subjects selected, 114 were controls and 124 were cases. All subjects had a physical examination. Weight and height were measured using standard techniques. BMI was calculated as weight $(\mathrm{kg})$ divided by the square of height $\left(\mathrm{m}^{2}\right)$.

\section{Sample Preparation}

Venipunctures were performed the morning after subjects had fasted for at least $8 \mathrm{~h}$. A minimally traumatic venipuncture was performed, using a 21-gauge butterfly needle, with the subject seated. After standardized processing at the clinical site, the samples were aliquoted into $2-\mathrm{ml}$ tubes. The samples were immediately centrifuged and the serum was stored at $-70^{\circ} \mathrm{C}$ until assayed.

\section{Hormone Assays}

Endogenous postmenopausal hormone status was assessed by measuring levels of estradiol, testosterone, SHBG and aromatase. Samples were analyzed at the Department of Clinical Laboratory at the Renmin Hospital of Wuhan University on an ACS-180 automated analyzer (Bayer Diagnostics, Tarrytown, N.Y., USA). Estradiol and testosterone were measured by competitive immunoassay using direct chemiluminescent immunoanalysis. Estradiol was assayed using rabbit anti- $\mathrm{E}_{2}-6$ ACS-180 immunoassay which was modified to reduce the lower limit of detection to $1.0 \mathrm{pg} / \mathrm{ml}$ and which had a coefficient of variation (CV) of 3-12\%. Testosterone was assayed using a polyclonal rabbit antitestosterone antibody and a monoclonal mouse anti-rabbit antibody coupled to paramagnetic particles. The testosterone-reporting range was $0.35-3.5 \mathrm{nM}(10-100 \mathrm{ng} / \mathrm{dl})$ and the assay range was $0.07-16.6 \mathrm{nM}$ $(2-478 \mathrm{ng} / \mathrm{dl})$. The inter- and intra-assay CV were 4.7 and $6.2 \%$, respectively. $10^{*} \mathrm{E}_{2} / \mathrm{T}$ was used to make the resulting ratio unit free [14]. SHBG and aromatase were measured with a commercial Enzyme Immunoassay kit purchased from Alpha Diagnostic International Inc. (San Antonio, Tex., USA). The total CV of SHBG ranged from 7.3 to $11.5 \%$ and the analytical sensitivity was $0.2 \mathrm{nM}$.

\section{Biochemical Assays}

Serum total cholesterol (TC), HDL-c, LDL-c, triglyceride (TG), glucose and high-sensitivity C-reactive protein (hsCRP) were assessed by an automatic biochemistry analyzer. Plasma levels of TC and TG were measured by enzymatic methods. The linear ranges of TC and TG were $0.5-18 \mathrm{mM}(20-700 \mathrm{mg} / \mathrm{dl})$ and $0.1-11.3 \mathrm{~mm}(10-1,000 \mathrm{mg} / \mathrm{dl})$, and the lowest limits of detection were 0.07 and $0.01 \mathrm{~mm}$, respectively. The total CV of TC ranged from 1.06 to $1.45 \%$, and that of TG was from 1.03 to $1.76 \%$. HDLc was also assessed enzymatically, and LDL-c was calculated using the Friedewald equation (LDL-c $=$ TC - TG/5 - HDL-c) [15]. The linear ranges of HDL-c and LDL-c were $0.05-4.65 \mathrm{mM}(2-180$ $\mathrm{mg} / \mathrm{dl})$ and $0.26-10.3 \mathrm{~mm}(10-400 \mathrm{mg} / \mathrm{dl})$, and the lowest limits of detection were 0.002 and $0.012 \mathrm{mM}$, respectively. The total CV of HDL-c ranged from 1.32 to $1.92 \%$, and that of LDL-c was from 2.34 to $2.71 \%$. Glucose was determined by the hexokinase method with the linear range from 0 to $22.24 \mathrm{mM}$. The inter- and intraassay $\mathrm{CV}$ were $\leq 6$ and $\leq 8 \%$, respectively. The atherogenic index of plasma (AIP), defined as the logarithm of the ratio TC:HDL-c, has recently been proposed as a biomarker of atherogenesis [16]. hsCRP was quantified using an immune turbidmetric analysis method. The lower limit of detection of the hsCRP assay was $0.03 \mathrm{mg} / \mathrm{dl}$, and the CV were $10-12 \%(0.05 \mathrm{mg} / \mathrm{dl})$ and $5-7 \%(2.2$ $\mathrm{mg} / \mathrm{dl})$.

\section{Statistical Analysis}

Statistical analysis was performed by the use of SPSS version 13.0. Variables were reported as means \pm SD. Skewed variables (SHBG, aromatase, hsCRP) were logarithmically transformed. Baseline characteristics, levels of sex hormones, aromatase, lipidlipoprotein profile and hsCRP were compared between the control and case groups using independent $t$ tests. The association between sex hormones and lipid parameters in cases was analyzed by multiple linear regression [17] after adjusting BMI and age. In addition, Pearson correlation between sex hormones, SHBG and aromatase was estimated in cases. Statistical significance was set at the 0.05 level. 


\section{Results}

\section{Baseline Characteristics}

As shown in table 1, there were no differences between postmenopausal patients with $\mathrm{CHD}$ and control postmenopausal women in terms of mean age, mean BMI, proportion of smokers and proportion of alcohol drinkers. However, sex hormone-related values had significant differences between controls and cases. Cases had a remarkable lower level of estradiol (18.53 \pm 7.55 in controls vs. $13.43 \pm 8.35$ in cases) but a higher level of testosterone (37.27 \pm 12.41 in controls vs. $38.33 \pm 15.02$ in cases) in comparison to controls. These measurements led to a significantly decreased $\mathrm{E}_{2} / \mathrm{T}$ ratio in cases $(5.35 \pm 2.78$ in controls vs. $3.88 \pm 2.51$ in cases, $\mathrm{p}<0.001)$. Compared with controls, there appears to be a lower level of serum aromatase $(2.23 \pm 0.48$ in controls vs. $2.11 \pm 0.32$ in cases $)$ and SHBG $(1.58 \pm 0.51$ in controls vs. $1.47 \pm 0.33$ in cases) in cases $(p=0.026$ and $p=0.042$, respectively). In the aspect of lipid-lipoprotein profile, TC levels showed no significant differences in the 2 groups. However, the concentration of HDL-c was lower in cases $(1.35 \pm 0.32$ in controls vs. $1.12 \pm 0.25$ in cases, $\mathrm{p}<0.001)$, and that of TG and LDL-c was higher $(1.15 \pm 0.41$ in controls vs. $1.65 \pm 0.83$ in cases for TG and $2.70 \pm 0.45$ in controls vs. $2.94 \pm 0.66$ in cases for LDL-c, all $\mathrm{p}<0.001)$. The AIP was higher in cases $(0.53 \pm 0.09$ in controls vs. $0.61 \pm 0.14$ in cases, $\mathrm{p}<0.001)$ and the HDL-c/LDL-c ratio was lower $(0.51$ \pm 0.14 in controls vs. $0.40 \pm 0.13$ in cases, $\mathrm{p}<0.001$ ) The hsCRP level increased markedly in women with CHD (0.14 \pm 0.34 in controls vs. $0.35 \pm 0.54$ in cases, $\mathrm{p}<0.001)$.

\section{Correlation of Sex Hormones with Lipoprotein Files}

Table 2 shows the results from multiple linear regression analysis between sex hormones and lipid parameters in 124 postmenopausal women with CHD. Because BMI and age might mediate the relation between hormones and carotid atherosclerosis, these variables were excluded from multivariate analysis. In cases, estradiol had a strong negative association with TC $(\mathrm{p}<0.001)$ and AIP $(\mathrm{p}<$ 0.001 ) while it had no statistical significance with TG, HDL-c, LDL-c or HDL-c/LDL-c. Testosterone showed significant positive associations with TC, HDL-c, AIP and HDL-c/LDL-c (p values 0.005, 0.002, <0.001 and $<0.001$, respectively).

In this study, according to the multiple linear regression analysis, the most striking observation is the correlation between the $\mathrm{E}_{2} / \mathrm{T}$ ratio and lipid profiles. The $\mathrm{E}_{2} / \mathrm{T}$
Table 1. Characteristics of controls and cases

\begin{tabular}{lccc}
\hline Characteristics & $\begin{array}{l}\text { Controls } \\
(\mathrm{n}=114)\end{array}$ & $\begin{array}{l}\text { Cases } \\
(\mathrm{n}=124)\end{array}$ & $\begin{array}{c}\mathrm{p} \\
\text { value }\end{array}$ \\
\hline Age, years & $64.00 \pm 8.70$ & $65.77 \pm 7.82$ & 0.499 \\
BMI & $22.00 \pm 1.72$ & $22.55 \pm 2.48$ & 0.550 \\
Smokers, n (\%) & $0(0)$ & $1(0.074)$ & 0.337 \\
Alcohol drinkers, n (\%) & $1(0.079)$ & $1(0.074)$ & 0.952 \\
Estradiol, pg/ml & $18.53 \pm 7.55$ & $13.43 \pm 8.35$ & $<0.001$ \\
Testosterone, ng/dl & $37.27 \pm 12.41$ & $38.33 \pm 15.02$ & 0.558 \\
E2/T & $5.35 \pm 2.78$ & $3.88 \pm 2.51$ & $<0.001$ \\
log(aromatase), nM & $2.23 \pm 0.48$ & $2.11 \pm 0.32$ & 0.026 \\
$\log ($ SHBG), nM & $1.58 \pm 0.51$ & $1.47 \pm 0.33$ & 0.042 \\
Total cholesterol, mM & $4.53 \pm 0.60$ & $4.54 \pm 0.87$ & 0.940 \\
Triglycerides, mM & $1.15 \pm 0.41$ & $1.65 \pm 0.83$ & $<0.001$ \\
HDL-c, mM & $1.35 \pm 0.32$ & $1.12 \pm 0.25$ & $<0.001$ \\
LDL-c, mM & $2.70 \pm 0.45$ & $2.94 \pm 0.66$ & 0.001 \\
AIP & $0.53 \pm 0.09$ & $0.61 \pm 0.14$ & $<0.001$ \\
HDL-c/LDL-c & $0.51 \pm 0.14$ & $0.40 \pm 0.13$ & $<0.001$ \\
Glucose, mM & $5.08 \pm 0.639$ & $5.46 \pm 1.528$ & 0.0563 \\
log (hsCRP), mg/dl & $0.14 \pm 0.34$ & $0.35 \pm 0.54$ & $<0.001$ \\
\hline
\end{tabular}

Mean value $\pm S D$. The independent $t$ test was used for comparison of continuous data and the $\chi^{2}$ test for proportions.

ratio was strongly related to lower TC, LDL-c and AIP but higher TG and HDL-c/LDL-c. In particular, the $\mathrm{E}_{2} / \mathrm{T}$ ratio had a negative association with AIP $(\mathrm{p}<0.001)$ and a positive association with HDL-c/LDL-c $(\mathrm{p}<0.001)$. SHBG showed a strong positive association with $\mathrm{TG}$ and TC among cases $(\mathrm{p}=0.027$ and $\mathrm{p}=0.033$, respectively). Aromatase was positively related to TC $(\mathrm{p}=0.013)$, LDL-c $(\mathrm{p}=0.043)$ and AIP $(\mathrm{p}=0.041)$ and was also inversely related to HDL-c/LDL-c $(\mathrm{p}=0.038)$.

Association between Sex Hormones and Aromatase or SHBG in Postmenopausal Women with CHD

The endogenous hormones were moderately or highly correlated with one another. Among the cases, the serum level of estradiol strongly predicted the $\mathrm{E}_{2} / \mathrm{T}$ ratio $(\mathrm{r}=$ $0.787, \mathrm{p}<0.001)$. Testosterone was negatively associated with the $\mathrm{E}_{2} / \mathrm{T}$ ratio $(\mathrm{r}=-0.383, \mathrm{p}<0.001)$ and positively associated with aromatase $(\mathrm{r}=0.268, \mathrm{p}=0.003)$. The $\mathrm{E}_{2} / \mathrm{T}$ ratio was inversely related with aromatase $(r=-0.192$, $\mathrm{p}=0.032$ ) and not significantly associated with SHBG; however, SHBG was highly correlated with aromatase $(\mathrm{r}=0.938, \mathrm{p}<0.001)$ (table 3$)$. 
Table 2. Multiple linear regression analysis of the relation between sex hormones and lipid parameters in 124 postmenopausal women with CHD

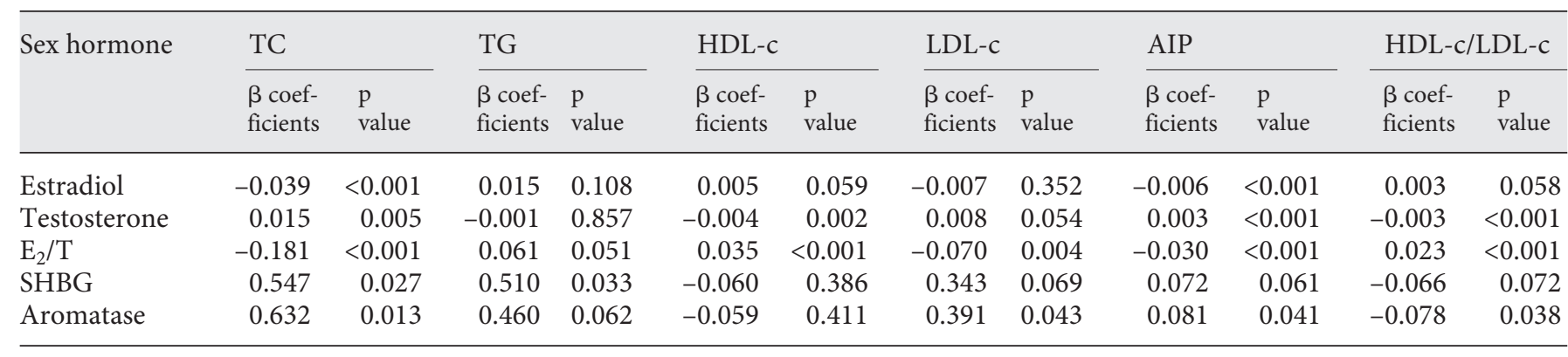

Results of multiple linear regression. Analysis adjusted for age and BMI.

Table 3. Correlation within SHBG, sex hormones and aromatase in postmenopausal women with CHD

\begin{tabular}{lrrrr}
\hline & $\begin{array}{l}\text { Estra- } \\
\text { diol }\end{array}$ & $\begin{array}{l}\text { Testos- } \mathrm{E}_{2} / \mathrm{T} \\
\text { terone }\end{array}$ & SHBG \\
& & & & \\
\hline $\begin{array}{l}\text { Testosterone } \\
\quad \text { Pearson correlation }\end{array}$ & 0.125 & & & \\
$\quad$ p value & 0.124 & & & \\
$\mathrm{E}_{2} / \mathrm{T}$ & & & & \\
$\quad$ Pearson correlation & 0.787 & -0.383 & & \\
$\quad$ p value & $<0.001$ & $<0.001$ & & \\
SHBG & & & & \\
$\quad$ Pearson correlation & -0.067 & 0.207 & -0.134 & \\
$\quad$ p value & 0.458 & 0.021 & 0.138 & \\
Aromatase & & & & \\
$\quad$ Pearson correlation & -0.086 & 0.268 & 0.192 & 0.938 \\
$\quad$ p value & 0.342 & 0.003 & 0.032 & $<0.001$ \\
\hline
\end{tabular}

\section{Discussion}

The relationship between sex hormones and CHD has been widely described. Estrogen has been associated with inflammatory processes, oxidative stress, angiogenesis and lipid metabolism, which indicated that it had beneficial vascular effects [18]. In a recent study focusing on the relationship between testosterone and lipoprotein in women, it was reported that testosterone was positively associated with lipid accumulation product and negatively associated with HDL-c and SHBG [19]. However, the association between the $\mathrm{E}_{2} / \mathrm{T}$ ratio and CHD is still unknown. One study has focused on the association between the $\mathrm{E}_{2} / \mathrm{T}$ ratio and disease. It explored the relationship between the balance of $\mathrm{E}_{2} / \mathrm{T}$ and the cerebral vascu- lature. It was found that altering the balance of estrogen and androgens can affect vascular tone, endothelial function, oxidative stress and inflammatory responses in cerebral vessels [20]. This indicated that keeping the balance of endogenous estrogen and androgen is extremely important in order to maintain homeostasis in the internal environment. We wondered, therefore, whether an imbalance of the $\mathrm{E}_{2} / \mathrm{T}$ ratio contributed most to the development of CHD in women.

In the present study, we found that the $\mathrm{E}_{2} / \mathrm{T}$ ratio was $5.35 / 1$ in the healthy postmenopausal women, while it was decreased to $3.88 / 1$ in the postmenopausal women with CHD. We further evaluated the relationship of an endogenous single sex hormone (estradiol or testosterone) or the $\mathrm{E}_{2} / \mathrm{T}$ ratio and the $\mathrm{CHD}$-related risk factors in postmenopausal women between controls and cases. Our results indicate that increasing the $\mathrm{E}_{2} / \mathrm{T}$ ratio was favorably associated with the cardiovascular lipid profile, suggesting that the $\mathrm{E}_{2} / \mathrm{T}$ ratio may be a more reliable parameter in evaluating cardiovascular risk.

A striking observation from this study is the association between the $\mathrm{E}_{2} / \mathrm{T}$ ratio and a panel of well-known risk factors for $\mathrm{CHD}$. It is clear that the $\mathrm{E}_{2} / \mathrm{T}$ ratio has a negative association with TC, LDL-c and AIP and a positive association with HDL-c and HDL-c/LDL-c. A previous study validated the significance of the AIP as an atherogenic marker [21]. These results suggest that the $\mathrm{E}_{2} / \mathrm{T}$ ratio could play an important role in modulating lipid profiles. This is the first report in demonstrating a negative association between the AIP and the $\mathrm{E}_{2} / \mathrm{T}$ ratio in postmenopausal women.

The $\mathrm{E}_{2} / \mathrm{T}$ ratio is mediated by several modulators in which the most important are aromatase and SHBG. Aromatase is the key enzyme for estrogen biosynthesis. It 
is responsible for the conversion of androgens to estrogens. We found a significantly positive association between the $\mathrm{E}_{2} / \mathrm{T}$ ratio and aromatase. This suggests that decreasing the concentration of aromatase may contribute to a lower conversion of androgens to estrogens; it may increase the level of testosterone and decrease the level of estradiol, thereby decreasing the $\mathrm{E}_{2} / \mathrm{T}$ ratio, resulting in its imbalance. We further investigated the relationship between aromatase and lipid profiles in postmenopausal women with $\mathrm{CHD}$ and found that aromatase was positively associated with TC, LDL-c and AIP and negatively associated with HDL-c/LDL-c. Aromatase may mediate its effect on the lipid profile by regulating the $E_{2} / T$ ratio. Other studies have provided proof that aromatase may play a role in outcomes among men and women with cardiovascular disease [22]. They have identified a striking association between CYP19A1 (encodes aromatase) genotype and outcomes in a group of postACS patients which was divergent in men and women. In women, the CYP19A1 -81371 C > T variant was associated with an increased risk of adverse outcomes; the variant was associated with a reduced risk for adverse outcomes. These findings could have broad implications for therapeutic usage by targeting aromatase.

SHBG is synthesized in the liver. It transports sex steroids and regulates the estrogen/androgen balance, thyroid hormones and insulin [14]. It binds up to $98 \%$ of the steroid hormones in the blood, including $5 \alpha$-dihydrotestosterone, testosterone and androstenediol with particularly high affinity, and estradiol and estrone with slightly lower affinity [23]. Thus, the bioavailability of sex hormones is influenced by the level of SHBG. Lower SHBG levels were shown in postmenopausal women with CHD. Studies have identified that SHBG levels were significantly correlated with intermediate $\mathrm{CHD}$ risk factor [24]. BMI is a major determinant of SHBG concentrations in women [25]. Therefore, the relationship between SHBG and lipid profile was done after adjusting BMI and age. In our study, in the postmenopausal women with CHD, SHBG was associated with a proatherogenic profile, which positively associated with TC and TG. However, some examinations of endogenous sex hormones and lipid levels in postmenopausal women may come to a different conclusion, i.e. that SHBG is associated with a more favorable lipid profile, including lower TC, LDL-c, TG and higher HDL-c among controls with minimal atherosclerosis [11, 26 , 27]. This association of SHBG with a more favorable lipid profile was not as prominent among cases with significant atherosclerosis. One explanation for the association between SHBG and lipid parameters is that the ef- fects of obesity and insulin resistance had not been taken into account. Another explanation is that SHBG may be favorable in healthy women, but unfavorable in postmenopausal women with CHD.

There have been varying reports about the role of androgen in postmenopausal women with CHD. Some data showed that increased androgenicity is associated with an increased risk of CHD [28]. Contrary to this assumption, other studies suggested that postmenopausal women with low testosterone levels have increased carotid atherosclerosis and an increased incidence of coronary events [8]. The same situation applies to the level of estradiol $[11,29,30]$. From the above data, we could consider that neither single estrogen nor single androgen could contribute to CHD development, and there must be a reasonable range of circulating estradiol and testosterone levels to maintain normal cardiovascular function [31]. Vitale et al. [32] held the same interpretation as us, since their study indicated that physiological androgens improve vascular functions and risk factors in women depending on background estrogen levels. Animal experiments resulted in the same observation: that the $\mathrm{E}_{2} / \mathrm{T}$ ratio, rather than the absolute levels of androgens or estrogens, is crucial in modulating the effect of sex hormones on atherosclerosis in female rabbits [33].

Certain limitations should be kept in mind when interpreting our data. Due to the limited sample size, we were unable to evaluate the joint effect of different sex hormones or the interaction of sex hormones and other lifestyle factors on CHD risk.

In conclusion, this study demonstrated that the $\mathrm{E}_{2} / \mathrm{T}$ ratio of serum levels is adversely altered in the postmenopausal women with CHD in the Chinese population. This imbalance is linked to an unfavorable cardiovascular risk profile, which suggests that maintenance of the balance of serum estradiol and testosterone levels may be critical in the treatment and prevention of CHD.

\section{Acknowledgements}

This study is supported by the Fundamental Research Funds for the Central University (grant No. 201130202020011) and the National Natural Science Foundation of China (grant No. 81100959).

\section{Conflict of Interest}

The authors have no conflict of interest. 


\section{References}

$>1$ Rappelli A: Hypertension and obesity after 12 Phillips GB, Pinkernell BH, Jing TY: Relathe menopause. J Hypertens Suppl 2002; 20:S26-S28.

2 Lambrinoudaki I, Christodoulakos G, Rizos $D$, et al: Endogenous sex hormones and risk factors for atherosclerosis in healthy Greek postmenopausal women. Eur J Endocrinol 2006;154:907-916.

-3 Chen Y, Zeleniuch-Jacquotte A, Arslan AA, et al: Endogenous hormones and coronary heart disease in postmenopausal women. Atherosclerosis 2011;216:414-419.

4 Karim R, Hodis HN, Stanczyk FZ, et al: Relationship between serum levels of sex hormones and progression of subclinical atherosclerosis in postmenopausal women. J Clin Endocrinol Metab 2008;93:131-138.

5 Manolio TA, Furberg CD, Shemanski L, et al: Associations of postmenopausal estrogen use with cardiovascular disease and its risk factors in older women. The CHS Collaborative Research Group. Circulation 1993;88: 2163-2171.

6 Golden SH, Maguire A, Ding J, et al: Endogenous postmenopausal hormones and carotid atherosclerosis: a case-control study of the atherosclerosis risk in communities cohort. Am J Epidemiol 2002;155:437-445.

7 Kumagai S, Kai Y, Sasaki H: Relationship between insulin resistance, sex hormones and sex hormone-binding globulin in the serum lipid and lipoprotein profiles of Japanese postmenopausal women. J Atheroscler Thromb 2001;8:14-20.

$>8$ Rexrode KM, Manson JE, Lee IM, et al: Sex hormone levels and risk of cardiovascular events in postmenopausal women. Circulation 2003;108:1688-1693.

$\checkmark 9$ Bernini GP, Sgro M, Moretti A, et al: Endogenous androgens and carotid intimal-medial thickness in women. J Clin Endocrino Metab 1999;84:2008-2012.

10 Reinecke H, Bogdanski J, Woltering A, et al: Relation of serum levels of sex hormone binding globulin to coronary heart disease in postmenopausal women. Am J Cardiol 2002;90:364-368.

11 Mudali S, Dobs AS, Ding J, et al: Endogenous postmenopausal hormones and serum lipids: the atherosclerosis risk in communities study. J Clin Endocrinol Metab 2005;90: 1202-1209. tionship between serum sex hormones and coronary artery disease in postmenopausal women. Arterioscler Thromb Vasc Biol 1997; 17:695-701.

13 Brown TM, Bittner V: Management of stable patients with coronary heart disease: clinical implications of the Clinical Outcomes Utilizing Revascularization and Aggressive Drug Evaluation (COURAGE) trial. J Clin Lipidol 2007;1:564-574.

14 Sowers MR, Randolph J Jr, Jannausch M, et al: Levels of sex steroid and cardiovascular disease measures in premenopausal and hormone-treated women at midlife: implications for the 'timing hypothesis'. Arch Intern Med 2008;168:2146-2153.

15 Friedewald WT, Levy RI, Fredrickson DS: Estimation of the concentration of low-density lipoprotein cholesterol in plasma, without use of the preparative ultracentrifuge. Clin Chem 1972;18:499-502.

-16 Tan MH, Johns D, Glazer NB: Pioglitazone reduces atherogenic index of plasma in patients with type 2 diabetes. Clin Chem 2004; 50:1184-1188.

17 Slinker BK, Glantz SA: Multiple linear regression: accounting for multiple simultaneous determinants of a continuous dependent variable. Circulation 2008;117:1732-1737.

18 Masood DE, Roach EC, Beauregard KG, et al: Impact of sex hormone metabolism on the vascular effects of menopausal hormone therapy in cardiovascular disease. Curr Drug Metab 2010;11:693-714.

19 Maturana MA, Moreira RM, Spritzer PM: Lipid accumulation product (LAP) is related to androgenicity and cardiovascular risk factors in postmenopausal women. Maturitas 2011;70:395-399.

20 Krause DN, Duckles SP, Gonzales RJ: Local oestrogenic/androgenic balance in the cerebral vasculature. Acta Physiol (Oxf) 2011; 203:181-186.

21 Dobiasova M: AIP - atherogenic index of plasma as a significant predictor of cardiovascular risk: from research to practice (in Czech). Vnitr Lek 2006;52:64-71.

22 Beitelshees AL, Johnson JA, Hames ML, et al: Aromatase gene polymorphisms are associated with survival among patients with cardiovascular disease in a sex-specific manner. PLoS One 2010;5:e15180.
23 Rosner W: Plasma steroid-binding proteins. Endocrinol Metab Clin North Am 1991;20: 697-720.

24 Sutton-Tyrrell K, Wildman RP, Matthews $\mathrm{KA}$, et al: Sex-hormone-binding globulin and the free androgen index are related to cardiovascular risk factors in multiethnic premenopausal and perimenopausal women enrolled in the Study of Women Across the Nation (SWAN). Circulation 2005;111:12421249.

25 de Moor P, Joossens JV: An inverse relation between body weight and the activity of the steroid binding globulin in human plasma. Steroidologia 1970;1:129-136.

26 Cauley JA, Gutai JP, Kuller LH, et al: The relation of endogenous sex steroid hormone concentrations to serum lipid and lipoprotein levels in postmenopausal women. Am J Epidemiol 1990;132:884-894.

27 Goodman-Gruen D, Barrett-Connor E: Sex hormone-binding globulin and glucose tolerance in postmenopausal women. The Rancho Bernardo Study. Diabetes Care 1997;20: 645-649.

28 Brand JS, van der Schouw YT: Testosterone, SHBG and cardiovascular health in postmenopausal women. Int J Impot Res 2010;22: 91-104.

-29 Bairey Merz CN, Johnson BD, Sharaf BL, et al: Hypoestrogenemia of hypothalamic origin and coronary artery disease in premenopausal women: a report from the NHLBIsponsored WISE study. J Am Coll Cardiol 2003;41:413-419.

30 Ossewaarde ME, Bots ML, van der Schouw YT, et al: Plasma and urinary sex hormones are differently related to lipids in healthy postmenopausal women. Maturitas 2003;44: 181-187.

31 Villablanca AC, Jayachandran M, Banka C: Atherosclerosis and sex hormones: current concepts. Clin Sci (Lond) 2010;119:493-513.

32 Vitale C, Mendelsohn ME, Rosano GM: Gender differences in the cardiovascular effect of sex hormones. Nat Rev Cardiol 2009; 6:532-542.

-33 Bruck B, Brehme U, Gugel N, et al: Genderspecific differences in the effects of testosterone and estrogen on the development of atherosclerosis in rabbits. Arterioscler Thromb Vasc Biol 1997;17:2192-2199. 